(C) 2017 IEEE. Personal use of this material is permitted. Permission from IEEE must be obtained for all other uses, in any current or future media, including reprinting/republishing this material for advertising or promotional purposes, creating new collective works, for resale or redistribution to servers or lists, or reuse of any copyrighted component of this work in other works. 


\title{
Study of Direct Coupling in Stator Dual Windings of Brushless Doubly-Fed Machine
}

\author{
Li Han, Xianpeng Ou, Jiang Du, Xuefeng Han, and Youguang Guo, Senior Member, IEEE
}

\begin{abstract}
A brushless doubly-fed machine (BDFM) usually contains two sets of independent three-phase symmetrical stator windings. Estimation and judgement of the direct electromagnetic coupling phenomenon between the two sets of stator windings is one of the most important prerequisites when safeguarding the excellent operation and control performances of the BDFM. However, because of the diversity among the stator winding structures, the generation mechanism and the existing direct coupling criterion between the two sets of stator windings have not been fully revealed to date. This paper analyzes the mutually-induced electromotive force (EMF) in the coils per pole per phase of a single stator winding that is induced by the rotating magnetic field of the other stator winding. Detailed studies of the effects of the winding structures on the mutuallyinduced EMF and the circulating current are performed and the results are verified by finite element analysis and experimental studies of an existing BDFM prototype. The results show that the direct coupling process between the two sets of stator windings is related to the combined number of poles, the number of parallel branches, the winding type and the winding connection.
\end{abstract}

Index Terms--Brushless doubly-fed machine (BDFM), stator winding, direct coupling, finite element analysis, experimental study.

\section{INTRODUCTION}

$\mathrm{B}$ RUSHLESS doubly-fed machines (BDFMs) have good potential for applications in the fields of variablefrequency-adjustable-speed motors and variable-speedconstant-frequency generators because they offer high reliability and low maintenance costs by virtue of the absence of brushes and slip rings in these machines [1], and because the associated inverter requires only a fractional rating of the machine. However, the BDFM structure is complex, and its efficiency and power-to-weight ratio are slightly lower than

This work was sponsored by the Visiting Scholarship Administration of the State Key Laboratory of Power Transmission Equipment \& System Security and New Technology (Chongqing University) under Grant No. 2007DA10512716403 and the State Key Laboratory of Advanced Electromagnetic Engineering and Technology (Huazhong University of Science and Technology) under Grant No. 2016KF001.

L. Han, X. Ou, J. Du, and X. Han are with the School of Electrical Engineering, Chongqing University, Chongqing 400044, China (e-mail: hanli@cqu.edu.cn; 20104449@cqu.edu.cn; 863829401@qq.com; 2313037904@qq.com).

Y. Guo is with the Faculty of Engineering and Information Technology, University of Technology Sydney, PO Box 123, Broadway NSW 2007, Australia (e-mail: youguang.guo-1@uts.edu.au). those of conventional ac machines with the same rating. A BDFM usually contains two sets of independent three-phase symmetrical stator windings with different numbers of poles, which are known as the power winding (PW) with a higher pole number and the control winding $(\mathrm{CW})$ with a lower pole number. The two sets of stator windings share a common stator magnetic circuit. Early studies usually considered that no direct electromagnetic coupling occurred between the two stator winding sets.

Electromagnetic coupling between the two stator windings can be achieved by modulation of the magnetic field of the rotor for electromechanical energy conversion [2], as shown in Fig. 1. However, if direct coupling exists between the two stator windings, an additional mutually induced electromotive force (EMF) could then be generated between the stator windings, and this will increase the stator winding voltage harmonics and cause a circulating current. This would not only increase the copper winding losses and reduce the output of the machine, but would also increase the complexity of the BDFM's dynamic mathematical model and the control difficulty [3]-[4]. It is therefore necessary to avoid generation of a mutually induced EMF between the two stator windings due to direct coupling as part of the machine design process, and estimation and judgement of the direct electromagnetic coupling phenomenon between the two stator windings is a significant task.

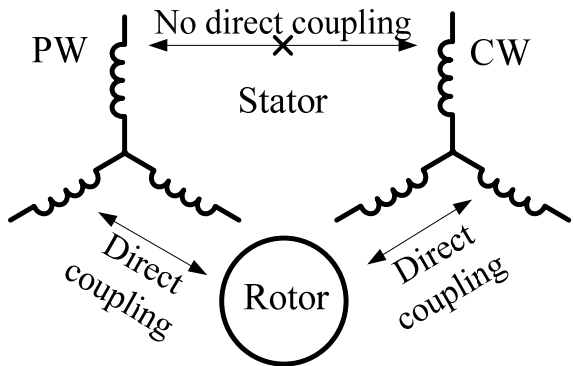

Fig. 1. Electromagnetic coupling in a BDFM.

Researchers have long believed that direct coupling between the two stator windings can be prevented by appropriate setting of the combination of pole numbers; general guidelines have been provided that the pole numbers of the two stator winding sets should be unequal, and the ratio of the pole numbers should meet the correlation conditions of being even multiples or an integer multiple of three [5]-[7]. However, existing BDFMs have many different pole number combinations, and their ratios not only meet the correlation conditions of being even multiples or an integer multiple of 
three, but also include other combinations such as $2 / 1,7 / 5,5 / 2$, and 6/4 [8]-[14]. Additionally, the effects of parallel branch numbers and different winding types on direct coupling between the two stator windings have not attracted sufficient attention, because these effects have rarely been reported. The complexity of the stator winding structures used means that direct coupling may still exist because of the different parallel branch numbers and other conditions, even if the pole number combination of the two stator windings is appropriate, and this results in additional mutually induced EMFs and circulating currents. In [15], for a $6 / 2$ pole combination BDFM with a salient-pole rotor, a dc component was found in the mutual inductance between the two stator windings; the component did not participate in the electromechanical energy conversion process, but the paper did not investigate the generation mechanism and its effects further. In [16], based on a calculation of the mutual inductance parameters between the two stator windings in a BDFM, principles for calculation of appropriate combinations of pole numbers were proposed to eliminate the direct coupling phenomenon, but no suitable simulated or experimental validation results were presented in support. Therefore, the direct coupling that occurs between the two stator windings is not explored fully, and further study is desirable.

This paper provides a detailed analysis of the mutually induced EMF in the coils per pole per phase of a single stator winding that is induced by the rotating magnetic field from the other stator winding. Further studies are performed to determine the effects of different pole number combinations, parallel branch numbers, winding types and winding connections on the mutually induced EMFs and circulating currents. The generation mechanism and the existing criterion of direct coupling between the two stator windings are revealed. Finally, the theoretical analysis results are verified using a finite element (FE) analysis and an experimental study of a BDFM prototype.

\section{Mutually INDUCED EMF IN STATOR WindingS}

For convenience in the analysis, the two stator windings of the BDFM are called winding 1 and winding 2 . All variables with subscript 1 represent the quantities of winding 1, while the variables with subscript 2 represent the quantities of winding 2 . The winding axis of phase $A$ is set as the origin of the spatial coordinates, and the arrangement sequence of phases $A-B-C$ represents the positive direction of the spatial mechanical angle.

Suppose that both stator windings are three-phase symmetrical, $60^{\circ}$ phase-belt, double-layer lap windings with integer numbers of slots and that they are independent from each other. The pole pair numbers are $p_{1}$ and $p_{2}$, and the associated parallel branch numbers are $a_{1}$ and $a_{2}$, respectively. The direct coupling relationship between the two stator windings can be judged based on whether or not the magnetic field generated by one stator winding causes a mutually induced EMF and a circulating current in the other stator winding.
The effects of saturation, slotting and harmonic fields are assumed to be negligible. When winding 1 produces an air gap rotating magnetic field that acts on winding 2 , the resulting field can be expressed in the stator coordinate frame as:

$$
b_{1}=\sqrt{2} B_{1} \cos \left(\omega_{1} t-p_{1} \theta\right)
$$

where $B_{1}$ is the root mean square (rms) value of the fundamental air gap flux densities of the $2 p_{1}$ poles, and $\omega_{1}$ is the angular frequency of winding 1.

The flux linkage that links a single coil of winding 2 because of $b_{1}$ can be expressed as follows:

$$
\begin{aligned}
\Psi_{2 \mathrm{c}} & =N_{2 \mathrm{c}} l D / 2 \int_{\theta_{2 \mathrm{c}}-\beta_{2 \mathrm{c}}}^{\theta_{2 \mathrm{c}}+\beta_{2 \mathrm{c}}} B_{1} \cos \left(\omega_{1} t-p_{1} \theta\right) \mathrm{d} \theta \\
& =N_{2 \mathrm{c}} l D B_{1} / p_{1} \sin \left(p_{1} \beta_{2 \mathrm{c}}\right) \cos \left(\omega_{1} t-p_{1} \theta_{2 \mathrm{c}}\right)
\end{aligned}
$$

where $N_{2 c}$ is the number of turns per coil of winding $2, l$ is the axial length of the stator core, $D$ is the inner diameter of the stator core, $\theta_{2 \mathrm{c}}$ is the coil axis position angle of winding 2 relative to the coordinate origin, and $\beta_{2 \mathrm{c}}$ is half of the coil span of winding 2.

The mutually induced EMF in a single coil and in a coil group per pole per phase of winding 2 are then given as follows, respectively:

$$
\begin{gathered}
e_{2 \mathrm{c}}=-\mathrm{d} \Psi_{2 \mathrm{c}} / \mathrm{d} t \\
=N_{2 \mathrm{c}} l D B_{1} \omega_{1} / p_{1} \sin \left(p_{1} \beta_{2 \mathrm{c}}\right) \sin \left(\omega_{1} t-p_{1} \theta_{2 \mathrm{c}}\right) \\
e_{2 \mathrm{q}}=q_{2} k_{2 \mathrm{~d}} e_{2 \mathrm{c}}=\sqrt{2} E_{2 \mathrm{q}} \sin \left(\omega_{1} t-p_{1} \theta_{2 \mathrm{c}}\right)
\end{gathered}
$$

where $q_{2}$ is the number of coils per pole per phase of winding $2, k_{2 \mathrm{~d}}$ is the winding distribution factor for the $p_{1} / p_{2}^{\text {th }}$ spatial harmonics, and $E_{2 q}$ is the rms value of the mutually induced EMF in the coil group per pole per phase of winding 2 .

\section{ANALYSIS OF THE DiReCt COUPLING}

\section{A. Effects of Pole Number and Parallel Branch Number}

Phase $A$ of winding 2 is arranged as shown in Fig. 2. Because the winding is supposed to be a double-layer lap winding, the number of coil groups per phase is equal to the number of poles, and the mechanical angular displacement between adjacent coil groups is $\pi / p_{2}$. All coil groups in phase $A$ are connected as shown in Fig. 3, where $k$ is a positive integer. The parallel branch number $a_{2}$ is less than or equal to the number of poles $2 p_{2}$, which is an integer multiple of $a_{2}$. When the rotating magnetic field of $2 p_{1}$ poles acts on winding 2 , the resulting phase angle difference between the mutually induced EMFs of the adjacent coil groups is $p_{1} \pi / p_{2}$.

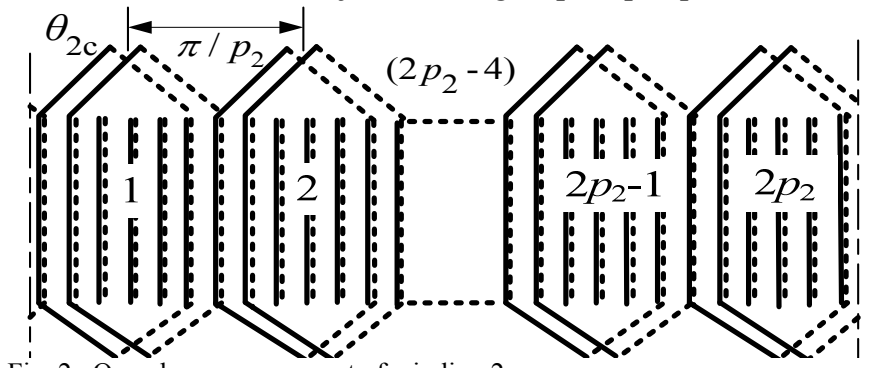

Fig. 2. One phase arrangement of winding 2. 


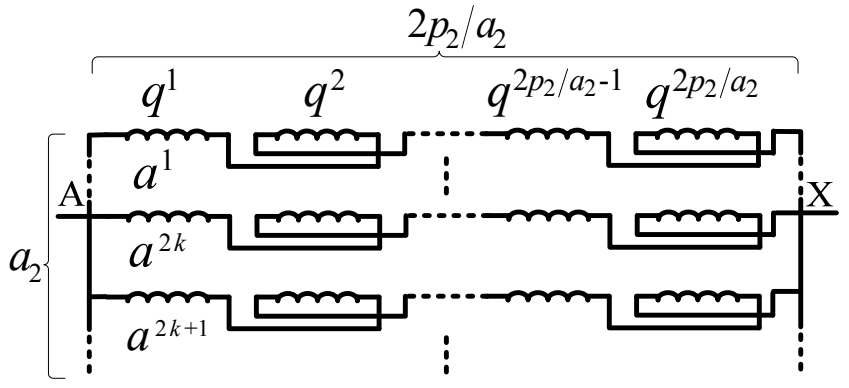

(a)

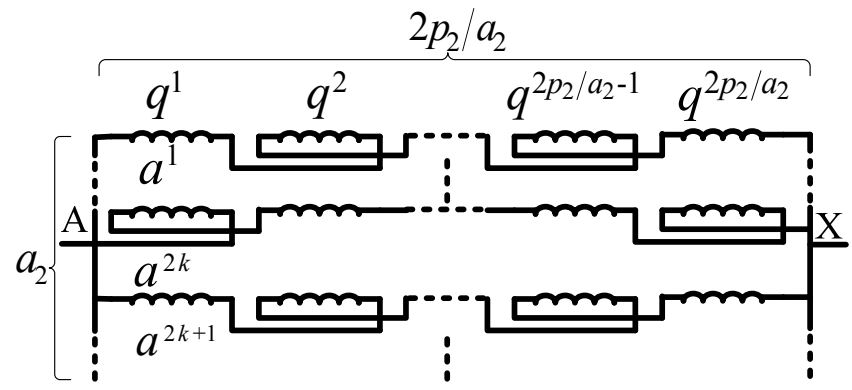

(b)

Fig. 3. Connections among coil groups in a single phase of winding 2 when (a) $2 p_{2} / a_{2}$ is even and (b) $2 p_{2} / a_{2}$ is odd.

Equation (4) shows that the amplitudes of the mutually induced EMFs in the various coil groups are equal. Because adjacent coil groups are connected in opposite directions, the resulting mutually induced EMF in the first branch $a^{1}$ of phase $A$ can be expressed as:

$$
e_{\mathrm{a}}=\sum_{j=1}^{2 p_{2} / a_{2}}(-1)^{j-1} E_{2 \mathrm{q}} \sin \left[\omega_{1} t-p_{1}\left(\theta_{2 \mathrm{c}}-\frac{j-1}{p_{2}} \pi\right)\right]
$$

where $j$ denotes the serial number of each of the coil groups per branch.

We define

$$
\begin{aligned}
& \alpha=\omega_{1} t-p_{1} \theta_{2 \mathrm{c}} \\
& \beta=p_{1} \pi / p_{2}
\end{aligned}
$$

Substitution of (6) and (7) into (5) then gives

$$
\begin{gathered}
e_{\mathrm{a}}=E_{2 \mathrm{q}}\{\sin \alpha-\sin (\alpha+\beta)+\sin (\alpha+2 \beta) \cdots \\
\left.+(-1)^{2 p_{2} / a_{2}-1} \sin \left[\alpha+\left(2 p_{2} / a_{2}-1\right) \beta\right]\right\}
\end{gathered}
$$

We then define $z=\cos \beta+\mathrm{i} \sin \beta$

$$
S=z-z^{2}+z^{3} \cdots+(-1)^{2 p_{2} / a_{2}}(z)^{2 p_{2} / a_{2}-1}
$$

When $j$ is a positive integer, then

$$
z^{j}=\cos j \beta+\mathrm{i} \sin j \beta
$$

Equation (10) can be changed into the following form:

$$
\begin{gathered}
S=\cos \beta-\cos 2 \beta \cdots+(-1)^{2 p_{2} / a_{2}} \cos \left[\left(2 p_{2} / a_{2}-1\right) \beta\right]+ \\
\mathrm{i}\left\{\sin \beta-\sin 2 \beta \cdots+(-1)^{2 p_{2} / a_{2}} \sin \left[\left(2 p_{2} / a_{2}-1\right) \beta\right]\right\}
\end{gathered}
$$

From (10) and (11), we obtain:

$$
S= \begin{cases}1-2 p_{2} / a_{2}, & z=-1 \\ {\left[z+(-z)^{2 p_{2} / a_{2}}\right] /(1+z),} & z \neq-1\end{cases}
$$

$$
(-z)^{2 p_{2} / a_{2}}=(-1)^{2 p_{2} / a_{2}}\left(\cos \frac{2 p_{1}}{a_{2}} \pi+\mathrm{i} \sin \frac{2 p_{1}}{a_{2}} \pi\right)
$$

Substitution of (12) into (8) then gives

$$
e_{\mathrm{a}}=E_{2 \mathrm{q}}[\sin \alpha-\sin \alpha \cdot \operatorname{Re}(S)-\cos \alpha \cdot \operatorname{Im}(S)]
$$

If $z=-1$, then $p_{1} / p_{2}=2 k-1$, and $S=1-2 p_{2} / a_{2}$. Therefore, the resulting mutually induced EMF can be expressed as:

$$
\begin{aligned}
& e_{\mathrm{a}}=E_{2 \mathrm{q}}\left[\sin \alpha-\sin \alpha \cdot\left(1-2 p_{2} / a_{2}\right)-\cos \alpha \cdot 0\right] \\
& =2 p_{2} / a_{2} E_{2 \mathrm{q}} \sin \left(\omega_{1} t-p_{1} \theta_{2 \mathrm{c}}\right)
\end{aligned}
$$

If $z \neq-1$, then $p_{1} / p_{2} \neq 2 k-1$, if and only if both $2 p_{2} / a_{2}$ and $2 p_{1} / a_{2}$ are odd or even, and then (13) and (14) can be given as

$$
\left\{\begin{array}{c}
(-z)^{2 p_{2} / a_{2}=1} \\
S=\left[z+(-z)^{2 p_{2} / a_{2}}\right] /(1+z)=1
\end{array}\right.
$$

As a result,

$$
e_{\mathrm{a}}=E_{2 \mathrm{q}}[\sin \alpha-\sin \alpha \cdot 1-\cos \alpha \cdot 0]=0
$$

The other branches of phase $A$ follow the same rules as branch $a^{1}$. When the mutually induced EMF is produced in the branch, the amplitudes of the EMFs in the adjacent branches are all equal. Each branch has $2 p_{2} / a_{2}$ coil groups, and the phase angle difference between the mutually induced EMFs of these adjacent coil groups is $p_{1} \pi / p_{2}$; this implies that for two coil groups with the same serial number between their adjacent branches, the phase angle difference of the mutually induced EMFs is given by $2 p_{1} \pi / a_{2}$.

Therefore, for adjacent branches, if $2 p_{2} / a_{2}$ is even (as shown in Fig. 3(a)), then the coil groups per branch are connected using the same pattern, and the phase angle difference is $2 p_{1} \pi / a_{2}$. If $2 p_{2} / a_{2}$ is odd, as shown in Fig. 3(b), then the coil groups of the two adjacent branches are connected in the opposite direction, and the phase angle difference is $2 p_{1} \pi / a_{2}+\pi$.

Phases $B$ and $C$ follow the same rules as phase $A$. Because the difference in the spatial mechanical angle for the threephase symmetrical winding axes is $2 \pi /\left(3 p_{2}\right)$ relative to each other, the amplitudes of the mutually induced EMFs of the three-phase windings are equal, but the phase angle differences are $2 p_{1} \pi /\left(3 p_{2}\right)$ relative to each other.

The following results can therefore be concluded.

When $p_{1} / p_{2}=2 k-1$, and if $2 p_{2} / a_{2}$ is even, the phase angle difference between the mutually induced EMFs of the adjacent branches is given by $2 p_{1} \pi / a_{2}=2 p_{2}(2 k-1) \pi / a_{2}$, which results in the mutually induced EMFs of the adjacent branches having the same phase angles; if $2 p_{2} / a_{2}$ is odd, then the phase angle difference is $2 p_{1} \pi / a_{2}+\pi$, and the phase angles remain the same. In this case, the mutually induced EMFs of the branches in phase $A$ are equal in both amplitude and phase, but the phase angles of the mutually induced EMFs of the three-phase windings are not equal unless $p_{1} / p_{2}=3 k$.

When $p_{1} / p_{2} \neq 2 k-1$, and if and only if both $2 p_{2} / a_{2}$ and $2 p_{1} / a_{2}$ are odd or even, no mutually induced EMFs can be produced in the winding. Otherwise, the mutually induced EMFs between the two branches or phase windings have the same amplitude but different phase angles. 


\section{B. Influence of Winding Type}

In addition to double-layer lap windings, ac machines use a variety of other winding types, including the double-layer wave winding, the single-layer chain winding, and concentric and cross windings. It is thus necessary to determine whether or not these winding types have the same rules as the doublelayer lap windings.

The EMF of the coil is relevant within the span and slot part of the coil, but is irrelevant within the coil end part. Because the double-layer wave winding structures are the same as those of the double-layer lap windings, apart from the coil end connection, the EMFs of the double-layer wave windings are the same as those of the double-layer lap windings. Therefore, the direct coupling relationship results for these two different double layer ac windings are the same.

For the single-layer concentric and chain windings, the coil groups are distributed evenly around the stator core and have the same coil numbers and spans. When one stator winding is considered to produce an air gap rotating magnetic field that acts on the other stator winding, then the mutually induced EMF follows the same rules as those for the double-layer lap windings.

For the single-layer cross windings shown in Fig. 4, the coil groups are distributed evenly around the stator core, but there are different coil numbers and spans between adjacent coil groups in each phase. Therefore, the phase angle differences of the mutually induced EMFs between these coils are equal, but the amplitudes of the mutually induced EMFs in adjacent coil groups differ when the rotating magnetic field of the $2 p_{1}$ poles acts on the single-layer cross winding. Therefore, there is no EMF difference between two branches or two phase windings if and only if $p_{1} / p_{2}$ is an integer multiple of three and $2 p_{2} / a_{2}$ is even.

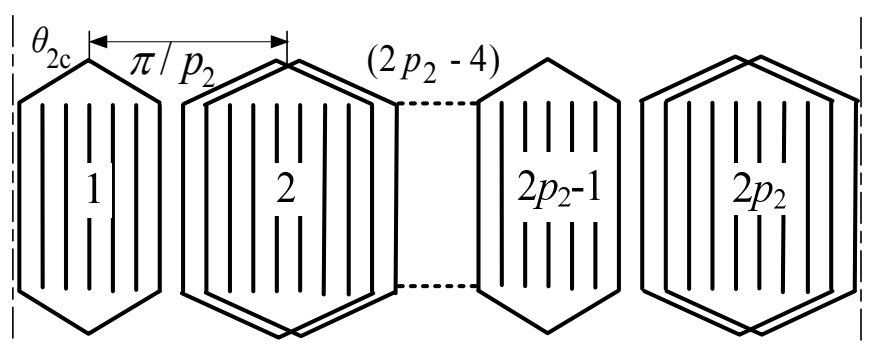

Fig. 4. Arrangement of single layer cross winding.

\section{Summary of Direct Coupling Principles}

The following principles can then be given to summarize the preceding discussion.

Direct coupling between the two stator windings is related to the number of poles, the number of parallel branches and the winding type. When winding 1 is considered to produce an air gap rotating magnetic field that acts on star-connected winding 2 without a neutral line, then the results for the winding types mentioned above are as follows. If winding 2 is a single-layer cross winding, then there is no direct coupling between the two stator windings only when $p_{1} / p_{2}$ is an integer multiple of three and $2 p_{2} / a_{2}$ is even. If winding 2 is not a single-layer cross winding, then:

1) $p_{1} / p_{2}$ is odd: the mutually induced EMFs in branches of the same phase are equal, without an EMF difference or a circulating current appearing between adjacent branches.

If $p_{1} / p_{2}$ is an integer multiple of three, then the mutually induced EMFs in the three-phase windings are equal in both amplitude and phase. Therefore, the circulating current and the direct coupling do not occur in winding 2. This result conforms to the general rules that EMF harmonics of the order of multiples of three would not appear in windings connected in a star configuration.

If $p_{1} / p_{2}$ is not an integer multiple of three, then the amplitudes of the mutually induced EMFs in the phase windings are equal, but the phase angles are not equal, then direct coupling exists.

2) $p_{1} / p_{2}$ is even or a non-integer: if and only if both $2 p_{2} / a_{2}$ and $2 p_{1} / a_{2}$ are either odd or even, the mutually induced EMF and direct coupling do not exist in the windings.

However, when winding 2 is connected in either a delta or a star configuration with a neutral line, then if and only if $p_{1} / p_{2}$ is even or a non-integer, both $2 p_{2} / a_{2}$ and $2 p_{1} / a_{2}$ are either odd or even; if winding 2 is not a single-layer cross winding, then the mutually induced EMF is not produced in the windings, and thus no direct coupling exists.

When the higher-order space harmonic $(v=5,7 \ldots)$ magnetic fields that are generated by the winding itself, slotting effects and core saturation are considered, the same analysis can be performed by substituting $v p_{1}$ for $p_{1}$, and the principles described above are still applicable.

\section{CASE ANALYSIS}

The summaries above are illustrated using the case of a BDFM prototype with the following parameters.

1) $P W: 4.5 \mathrm{~kW}$, six poles, double-layer lap winding, 12 series-connected coils per phase;

2) $C W: 1.5 \mathrm{~kW}$, two poles, single-layer concentric winding, six series-connected coils per phase;

3) Stator: 36 slots for both the star-connected PW and CW without neutral lines;

4) Rotor: 44 slots with a cage bar.

The diagrams for phase $A$ in the two stator windings are shown in Fig. 5. For convenience of both comparison and verification, it is redefined here that the variables with a subscript $p$ denote the PW quantities, and those with a subscript $c$ denote the $\mathrm{CW}$ quantities.

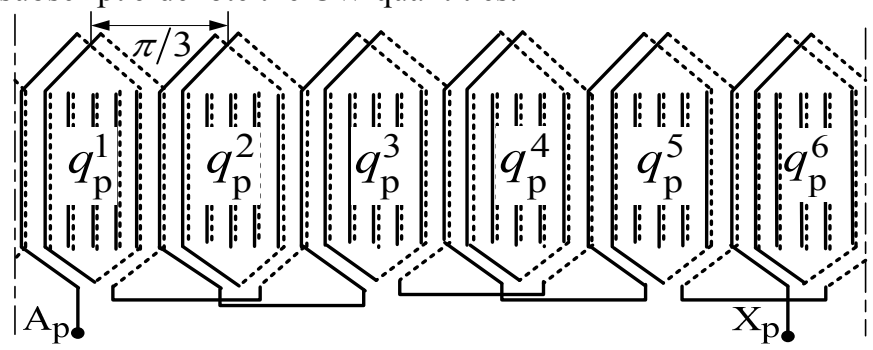

(a) 


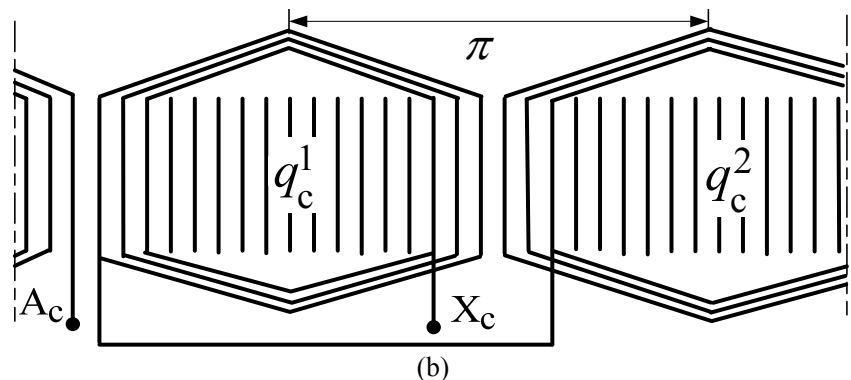

Fig. 5. Phase $A$ diagrams for (a) $\mathrm{PW}$ and (b) $\mathrm{CW}$.

When the PW is considered to produce a rotating magnetic field that acts on the $\mathrm{CW}$, then the mutually induced EMF in coil group $q_{\mathrm{c}}^{1}$ of phase $A$ can be derived from (4) as follows:

$$
e_{q_{\mathrm{c}}^{1}}=\sqrt{2} E_{\mathrm{cq}} \sin \left(\omega_{\mathrm{p}} t-3 \theta_{q_{\mathrm{c}}}\right)
$$

where $\theta_{q_{\mathrm{c}}^{1}}$ is the angular displacement of the $\mathrm{CW}$ coil group $q_{\mathrm{c}}^{1}$ axis from the coordinate origin, and $E_{\mathrm{cq}}$ is the rms value of the mutually induced EMF in each coil group per pole per phase of the $\mathrm{CW}$.

Using a phasor representation, the mutually induced EMFs in coil groups $q_{\mathrm{c}}^{1}$ and $q_{\mathrm{c}}^{2}$ can be expressed, respectively, as:

$$
\begin{gathered}
\dot{E}_{q_{\mathrm{c}}^{1}}=E_{\mathrm{cq}} \angle 3 \theta_{q_{\mathrm{c}}^{1}} \\
\dot{E}_{q_{\mathrm{c}}^{2}}=E_{\mathrm{cq}} \angle\left(3 \theta_{q_{\mathrm{c}}^{1}}-3 \pi\right)
\end{gathered}
$$

Because the connections between adjacent coil groups in a single phase are reversed, and because the spatial electrical angle difference between adjacent axes of the three-phase symmetrical winding axes is $2 \pi / 3$, the mutually induced EMF of the $\mathrm{CW}$ can be written as

$$
\left\{\begin{array}{l}
\dot{E}_{\mathrm{c} A}=\dot{E}_{q_{\mathrm{c}}^{1}}-\dot{E}_{q_{\mathrm{c}}^{2}}=2 E_{\mathrm{cq}} \angle 3 \theta_{q_{\mathrm{c}}^{1}} \\
\dot{E}_{\mathrm{c} B}=2 E_{\mathrm{cq}} \angle\left(3 \theta_{q_{\mathrm{c}}^{1}}-2 \pi\right) \\
\dot{E}_{\mathrm{c} C}=2 E_{\mathrm{cq}} \angle\left(3 \theta_{q_{\mathrm{c}}^{1}}+2 \pi\right)
\end{array}\right.
$$

Here, subscripts $A, B$, and $C$ denote the three phases. Equation (22) shows that the amplitudes and the phase angles of the mutually induced EMFs in the $\mathrm{CW}$ are equal. Because the $\mathrm{CW}$ is star-connected without a neutral line, the circulating current could not be produced in the CW.

When the $\mathrm{CW}$ produces a rotating magnetic field that acts on the PW, then, similarly, the mutually induced EMF in coil group $q_{\mathrm{p}}^{1}$ of phase $A$ is given by:

$$
\dot{E}_{q_{\mathrm{p}}^{1}}=E_{\mathrm{pq}} \angle 0
$$

where $E_{\mathrm{pq}}$ is the rms value of the mutually induced EMF in each coil group per pole per phase of the PW.

For the PW, the phase angle difference and the phasor diagram of the mutually induced EMF in the coil groups of phase $A$ are given in Table I and in Fig. 6 respectively, where the negative sign of $\dot{E}_{q_{\mathrm{p}}^{j}}$ indicates the reverse connection of the adjacent coil groups. Therefore, the synthetic mutually induced EMF in phase $A$ is zero; the same results apply for both phases $B$ and $C$.

TABLE I

Phase Angle Differences of Mutually InduCEd EMFS IN COIL GROUPS OF THE PW

\begin{tabular}{ccccccc}
\hline \hline Coil group & $q_{\mathrm{p}}^{1}$ & $q_{\mathrm{p}}^{2}$ & $q_{\mathrm{p}}^{3}$ & $q_{\mathrm{p}}^{4}$ & $q_{\mathrm{p}}^{5}$ & $q_{\mathrm{p}}^{6}$ \\
\hline \hline Phase angle difference & 0 & $\pi / 3$ & $2 \pi / 3$ & $\pi$ & $4 \pi / 3$ & $5 \pi / 3$ \\
\hline & & & \\
& & & \\
\hline
\end{tabular}

Fig .6. Phasor diagram of mutual induced EMFs in coil groups of the PW.

In the BDFM prototype described above, all coils per phase of each of the two stator windings are connected in series. The parallel branch numbers are now changed to verify that the analyses are correct. Because the number of poles of the PW and the $\mathrm{CW}$ are 6 and 2, respectively, the numbers of parallel branches $a_{\mathrm{p}}$ can be $1,2,3$, or 6 , while $a_{\mathrm{c}}$ can be 1 or 2 . From (20), (21) and Fig. 6, when one stator winding produces a rotating magnetic field that acts on the other stator winding, the analysis results for the mutually induced EMFs are as shown in Table II, in which $Z_{\mathrm{q}}$ is the impedance of a coil group. As a result:

1) The $P W$ produces a rotating magnetic field that acts on the $C W: p_{\mathrm{p}} / p_{\mathrm{c}}=3, a_{\mathrm{c}}=1,2$, and thus a mutually induced EMF exists within the branch. However, the EMFs that are mutually induced in the adjacent branches are equal in both amplitude and phase angle, and thus no circulating current exists, which conforms to summary 1), as given in Section III.

2) The $C W$ produces a rotating magnetic field that acts on the $P W$ : when $p_{\mathrm{c}} / p_{\mathrm{p}}=1 / 3$, and if $a_{\mathrm{p}}=1$ or 2 , then both $2 p_{\mathrm{c}} / a_{\mathrm{p}}$ and $2 p_{\mathrm{p}} / a_{\mathrm{p}}$ are odd or even, and the mutually induced EMFs in a branch are equal to zero; if $a_{\mathrm{p}}=3$ or $6,2 p_{\mathrm{c}} / a_{\mathrm{p}}$ and $2 p_{\mathrm{p}} / a_{\mathrm{p}}$ are not both odd or both even, then the mutually induced EMFs in adjacent branches are different, which results in a circulating current and thus conforms to summary 2) in Section III.

$$
\text { TABLE II }
$$

ANALYSIS RESULTS FOR MUTUALLY INDUCED EMF AND CirCUlating CURRENT IN A BRANCH

\begin{tabular}{ccccccc}
\hline \hline & \multicolumn{2}{c}{$\mathrm{CW}$} & \multicolumn{4}{c}{$\mathrm{PW}$} \\
\hline Parallel branch numbers & 1 & 2 & 1 & 2 & 3 & 6 \\
Mutual induced EMF & $2 E_{\mathrm{cq}}$ & $E_{\mathrm{cq}}$ & 0 & 0 & $E_{\mathrm{pq}}$ & $E_{\mathrm{pq}}$ \\
Circulating current & - & 0 & - & 0 & $E_{\mathrm{pq}} / 2 \mathrm{Z}_{\mathrm{q}} E_{\mathrm{pq}} / \mathrm{Z}_{\mathrm{q}}$ \\
\hline \hline
\end{tabular}

\section{FE ANALYSIS AND EXPERIMENTS}

The theoretical analysis is verified using FE analysis and experiment studies of the BDFM prototype described in Section IV, while taking the effects of the pole number combinations and the numbers of parallel branches on the direct coupling into account. The experimental platform and 
the prototype structure are shown in Figs. 7 and 8, that the theoretical analysis is correct.

respectively.

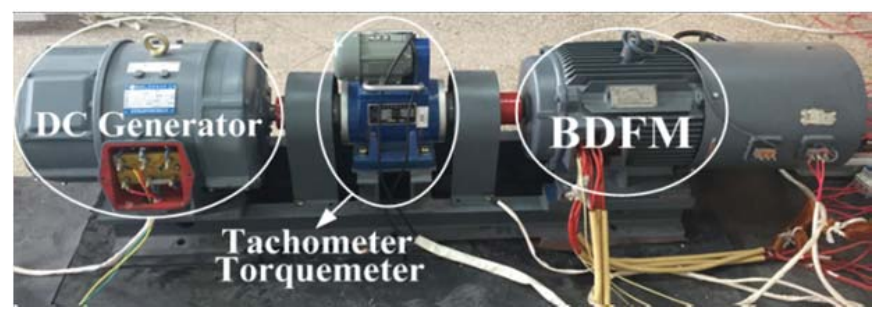

Fig. 7. Experimental platform.

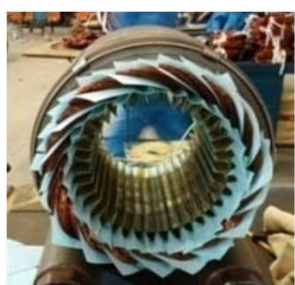

(a)

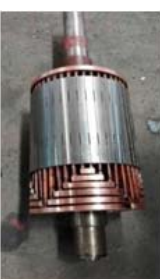

(b)
Fig. 8. (a) Stator and (b) rotor structures of BDFM prototype.

Using FLUX software, the FE analysis is executed on the 2D transient magnetic field. The FE analysis region of the BDFM prototype is as shown in Fig. 9. Because of the asymmetrical magnetic field distribution within the BDFM, the entire prototype region is set as the analysis area. The $z$ axis component of the magnetic vector potential is zero on the stator core's outer surface and the rotor core's internal surface because of the homogeneous boundary conditions.

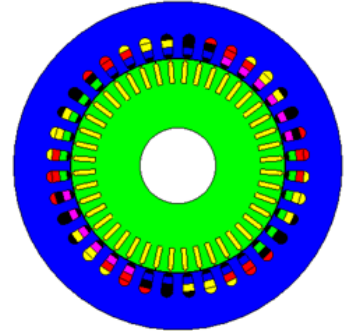

Fig. 9. FE analysis region of the BDFM prototype.

\section{A. Validation of the Effects of Pole Number Combinations}

1) Mode without Rotor: The rotor is pulled out to eliminate its effects during testing, and the rotor region in the FE model is set to be air. One stator winding is in turn supplied at 30 $\mathrm{V}_{\mathrm{rms}} / 50 \mathrm{~Hz}$ while the other stator winding remains opencircuited to enable observation and analysis of the mutually induced EMF waveforms within it.

When the PW is excited and the $\mathrm{CW}$ is open-circuited, the mutually induced EMFs in the $\mathrm{CW}$ are equal in both amplitude and phase angle, as Fig. 10 shows. When the $\mathrm{CW}$ is excited and the PW is open-circuited, then the mutually induced EMFs in the PW are nearly zero, as shown in Fig. 11.

For the prototype with stator windings with the $6 / 2$ poles, the results of the FE analysis and the experiments show that the fundamental magnetic field generated by the PW will cause the same mutually induced EMFs in the CW, but no mutually induced EMF exists in the PW when the fundamental magnetic field of the $\mathrm{CW}$ is acting upon it, and this verifies

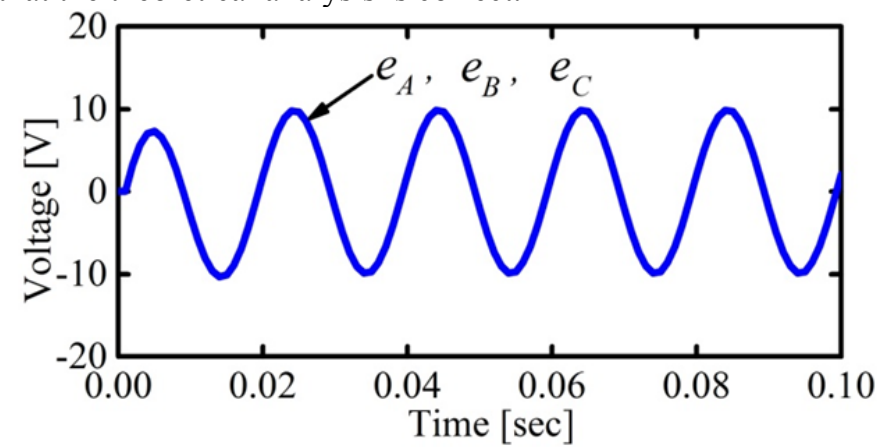

(a)

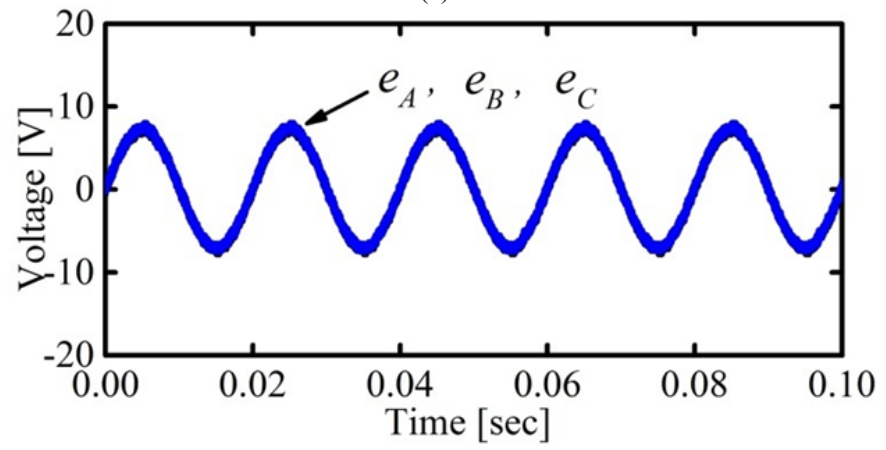

(b)

Fig. 10. (a) FE analysis and (b) test waveforms of three-phase mutually induced EMFs in the $\mathrm{CW}$.
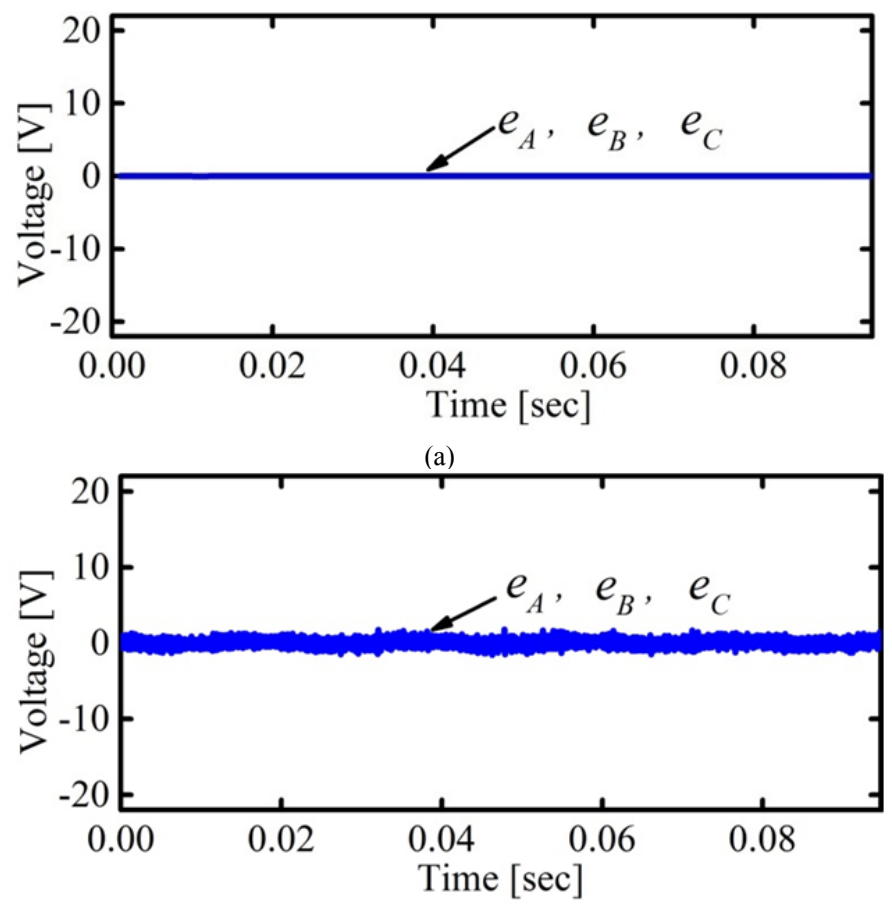

(b)

Fig. 11. (a) FE analysis and (b) test waveforms of three-phase mutually induced EMFs in the PW.

2) Mode with Motor: The prototype now operates as a normal motor, the PW is supplied at $220 \mathrm{~V}_{\mathrm{rms}} / 50 \mathrm{~Hz}$, and the $\mathrm{CW}$ is fed with a reverse-sequence voltage of $30 \mathrm{~V}_{\mathrm{rms}} / 5 \mathrm{~Hz}$. In this case, the prototype operates at an under-synchronous condition with a rotor speed of $675 \mathrm{r} / \mathrm{min}$. In the FE analysis, the time step is set to $0.001 \mathrm{~s}$, and the two stator windings are 
excited using ideal voltage sources. In the experiments, the $\mathrm{PW}$ is connected to the grid and the $\mathrm{CW}$ is supplied using an inverter. The results from the FE analysis and the experiments are shown in Figs. 12 and 13, respectively, where $U_{A B}$ represents the line-to-line voltage and $U_{A}$ represents the phase voltage.

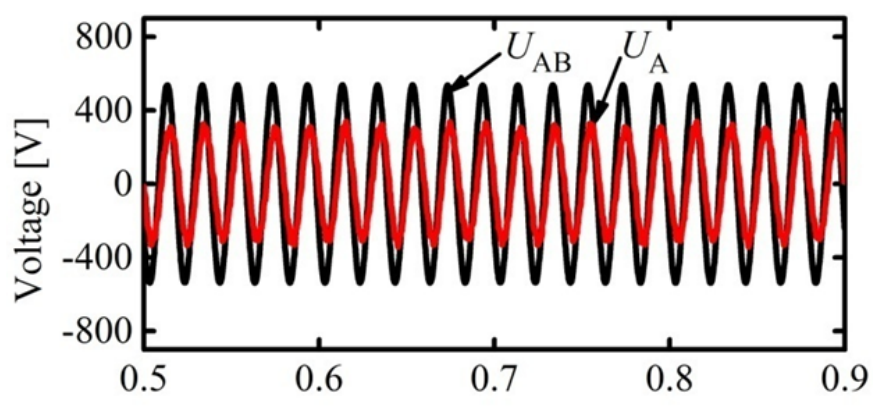

(a)

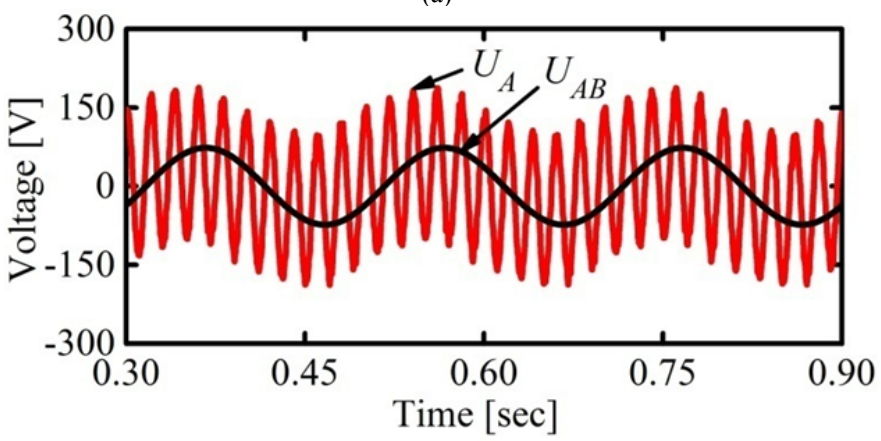

(b)

Fig. 12. FE results for phase and line-to-line voltages: (a) PW and (b) CW.

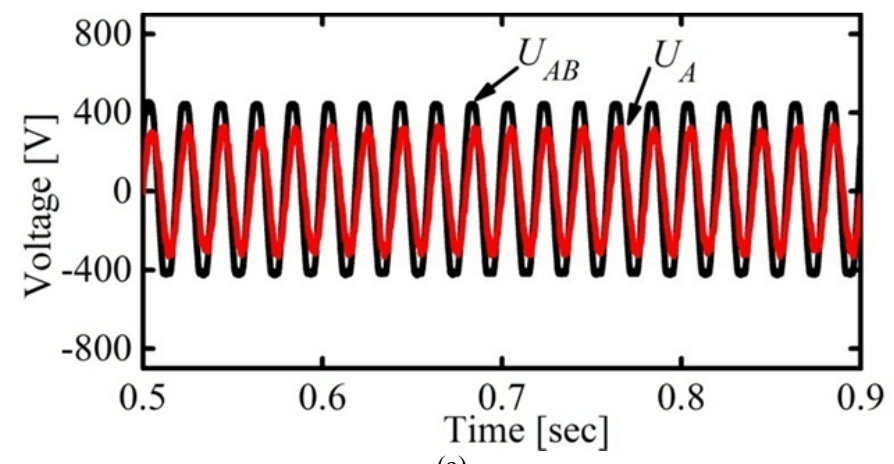

(a)

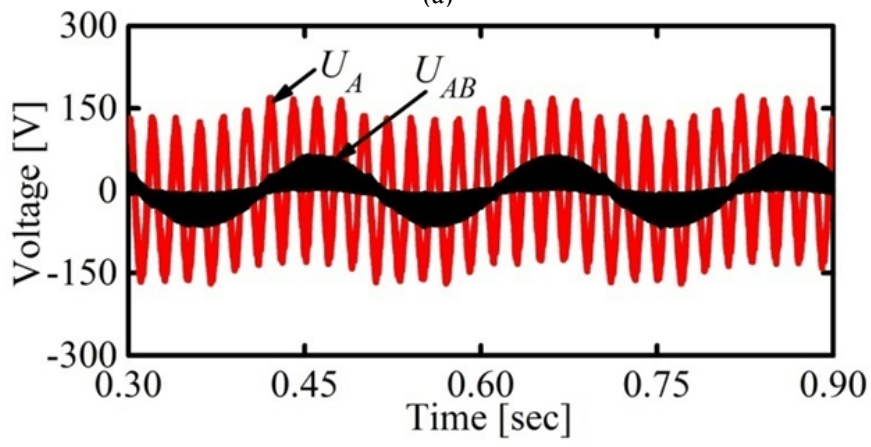

(b)

Fig. 13. Experimental results for phase and line-to-line voltages: (a) PW and (b) $\mathrm{CW}$.

Figs. 12 and 13 show that these voltages contain an abundance of harmonics. For ease of comparison, only the relevant harmonic components are indicated in the spectrum analysis, as shown in Fig. 14.

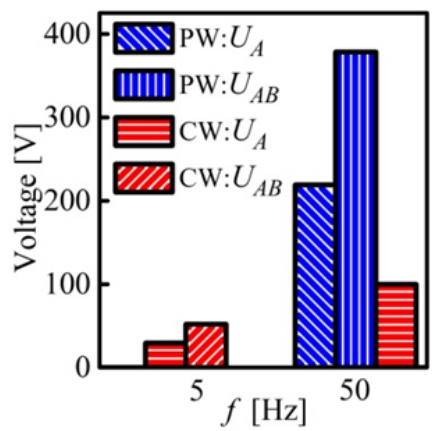

(a)

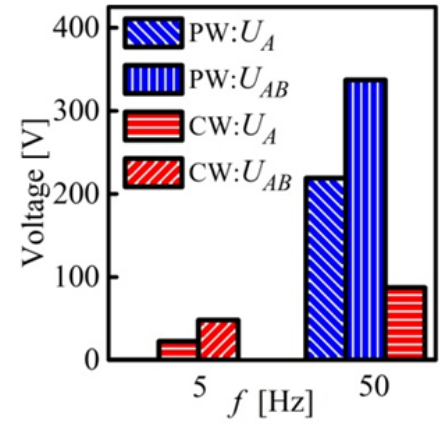

(b)
Fig .14. (a) FE analysis and (b) test comparisons of spectrum analyses of the phase and line-to-line voltages for the two stator windings.

Fig. 14 shows that the phase and the line-to-line voltages in the PW consist solely of $50 \mathrm{~Hz}$ self-induced EMFs. However, in the $\mathrm{CW}$, the phase voltage contains not only the $5 \mathrm{~Hz}$ selfinduced EMF, but also the $50 \mathrm{~Hz}$ mutually induced EMF due to direct coupling; the amplitude of the latter is high because it is proportional to the supply frequency and is inversely proportional to the number of pole pairs, as shown in (3). Because the amplitudes and the phase angles are equal for the three-phase mutually induced EMFs in the $\mathrm{CW}$ that were caused by the magnetic field of the $\mathrm{PW}$, the line-to-line voltage in the $\mathrm{CW}$ only contains the $5 \mathrm{~Hz}$ self-induced $\mathrm{EMF}$, and not the $50 \mathrm{~Hz}$ mutually induced EMF.

\section{B. Validation of the Effects of Parallel Branch Numbers}

For ease of analysis and comparison, the FE is analyzed using the model without the rotor. One stator winding is supplied at $100 \mathrm{~V}_{\mathrm{rms}} / 50 \mathrm{~Hz}$ while the other stator winding is open-circuited, and the numbers of parallel branches are varied.

When the PW is excited and the $\mathrm{CW}$ is open-circuited, the number of parallel branches $a_{\mathrm{c}}$ may be either 1 or 2 . When the $\mathrm{CW}$ is excited and the PW is open-circuited, the parallel branch numbers $a_{\mathrm{p}}$ may be $1,2,3$, or 6 .

Table III lists the FE analysis results for the mutually induced EMFs and the circulating currents for the different parallel branch numbers. For ease of understanding, the circuit of the stator windings and the circulating current waveforms in the branches of one phase in the PW are shown in Figs. 15 and 16 , respectively, when the $\mathrm{CW}$ is excited and $a_{\mathrm{p}}=3$.

TABLE III

FE ANALYSIS RESULTS FOR MUTUALLY INDUCED EMF AND CIRCULATING CURRENT IN A BRANCH

\begin{tabular}{ccccccc}
\hline \hline & \multicolumn{3}{c}{ CW } & \multicolumn{5}{c}{ PW } \\
\hline Parallel branch numbers & 1 & 2 & 1 & 2 & 3 & 6 \\
Mutual induced EMF (V) & 26.8 & 12.8 & 0 & 0 & 3.8 & 3.8 \\
Circulating current (A) & - & 0 & - & 0 & 4.4 & 8.1 \\
\hline \hline
\end{tabular}




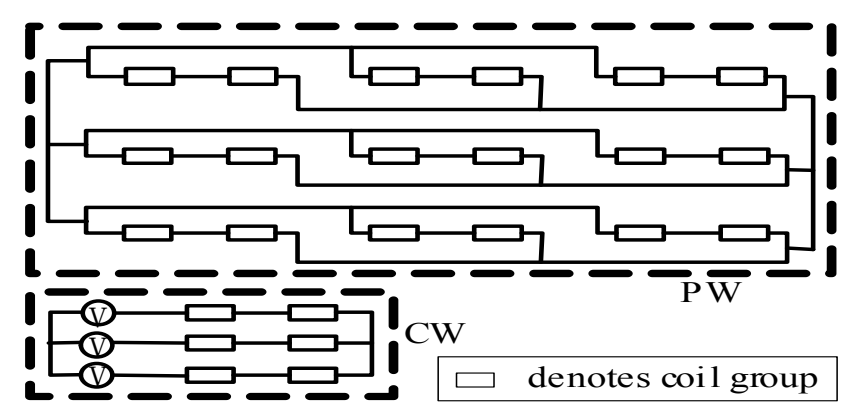

Fig. 15. Circuit of stator windings with $a_{\mathrm{p}}=3$.

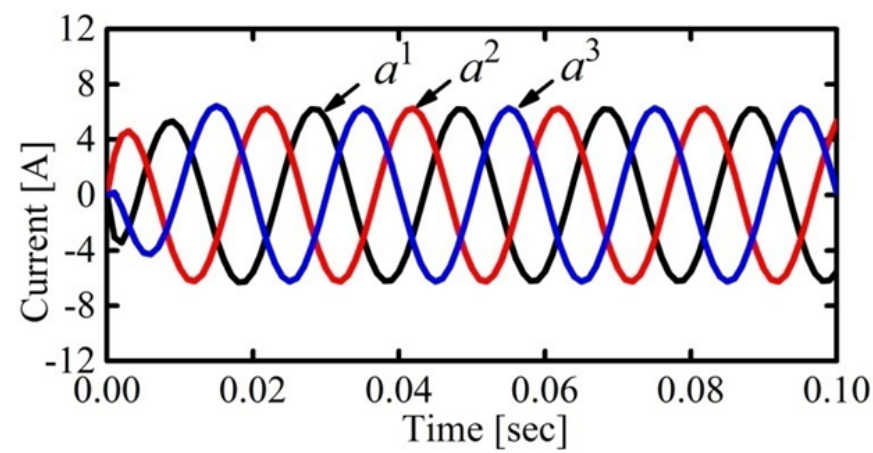

Fig. 16. Branch circulating current in one phase of PW with $a_{\mathrm{p}}=3$.

Table III shows that when the PW produces a rotating magnetic field that acts on the $\mathrm{CW}$, and when $a_{\mathrm{c}}=1$ or 2, the mutually induced EMFs then exist in the branches of the CW, but the EMF phase angles between the two branches are equal, so no circulating current is produced. When the $\mathrm{CW}$ produces a rotating magnetic field that acts on the PW, and when $a_{\mathrm{p}}=1$ or 2, no mutually induced EMF or circulating current would be produced in the branches; if $a_{\mathrm{p}}=3$, then the mutually induced EMF does occur in a branch and causes a circulating current with an amplitude of $4.4 \mathrm{~A}_{r m s}$, where the phase angle difference in the circulating current between the two branches is $2 \pi / 3$. If $a_{\mathrm{p}}=6$, then the mutually induced EMF exists in one branch, and the circulating current amplitude is $8.1 \mathrm{~A}_{r m s}$, which is nearly twice that produced when $a_{\mathrm{p}}=3$. The ratio relationship coincides with the analysis results shown in Table II, and any deviations may be caused by the saturation of the stator core. Additionally, for a stator winding with different parallel branch numbers, the amplitudes of the mutually induced EMFs in the branches also meet the same basic ratio relationship as that shown in Table II, which illustrates the results of the case analyses in Section IV.

Therefore, when $a_{\mathrm{p}}=3$ or 6 , the mutually induced EMFs in the $\mathrm{PW}$ branches will be produced by the rotating magnetic field of the $\mathrm{CW}$, which causes the circulating current, and the direct electromagnetic coupling phenomenon occurs between the two stator windings. The FE analysis results and the experimental results validate the theoretical analyses.

\section{CONCLUSIONS}

Based on theoretical analyses, FE simulations and experimental studies, this paper reveals the generation mechanism and existing criterion for direct coupling between the two stator windings of a BDFM with a cage rotor. The results show that direct coupling between the two stator windings is not only related to the pole number combinations, but is also related to the parallel branch numbers, the winding type and the winding connections. When winding 1 produces a rotating magnetic field that acts on the star-connected winding 2 without a neutral line, no direct coupling occurs under the following three conditions:

1) Winding 2 is not a single-layer cross winding, $p_{1} / p_{2}$ is odd, and $p_{1} / p_{2}$ is an integer multiple of three;

2) Winding 2 is not a single-layer cross winding, $p_{1} / p_{2}$ is even or a non-integer, and both $2 p_{2} / a_{2}$ and $2 p_{1} / a_{2}$ are odd or even; and

3) Winding 2 is a single-layer cross winding, $p_{1} / p_{2}$ is an integer multiple of three, and $2 p_{2} / a_{2}$ is even.

When winding 2 is connected in a delta or star configuration with a neutral line, and if and only if winding 2 is not a single-layer cross winding, $p_{1} / p_{2}$ is even or a noninteger, and both $2 p_{2} / a_{2}$ and $2 p_{1} / a_{2}$ are odd or even, then direct coupling does not exist. 